\title{
Circ-TTC17 Promotes Proliferation and Migration of Esophageal Squamous Cell Carcinoma
}

\author{
Qian Wang ${ }^{1} \cdot$ Qian Zhang ${ }^{1,2} \cdot$ Handong Sun ${ }^{1} \cdot$ Weiwei Tang ${ }^{3} \cdot$ Lili Yang $^{4} \cdot$ Zhipeng Xu $^{2} \cdot$ Zhihua Liu $^{5} \cdot$ Hui Jin $^{6,7,8}$. \\ Xiufeng $\mathrm{CaO}^{1,9}$
}

Received: 11 August 2018 / Accepted: 14 November 2018 / Published online: 5 December 2018

(c) The Author(s) 2018

\begin{abstract}
Background Circular RNAs (circRNAs), a special class of noncoding RNAs with the characteristic of covalent closed-loop structure, have been widely found in various organisms. Growing evidence has shown that circRNAs play a crucial role in regulating biological functions of cancers. However, the specific role of circRNAs in esophageal squamous cell carcinoma (ESCC) remains largely unknown.

Aim The present study aims to investigate the effects of circ-TTC17 in ESCC clinical samples as well as cells.

Methods Sanger sequencing and agarose gel electrophoresis were used to verify the specificity of circ-TTC17. Expression levels of circ-TTC17 in ESCC cells, plasma, and tissues were measured by quantitative real-time polymerase chain reaction. A colony formation experiment, CCK-8 assay, and wound-healing assay were applied to detect the functions of circ-TTC17 in KYSE30 and KYSE450 cells. A nucleus-cytoplasm fractionation experiment was used to probe the location of circ-TTC17 in ESCC cells. Finally, a network of circ-TTC17 with its targeted miRNAs interactions and corresponding mRNAs was analyzed and framed by bioinformatics.

Results The expression level of circ-TTC17 was found to be significantly higher in ESCC cells, plasma, and tissues compared with normal cases. In vitro experiments indicated that circ-TTC17 promoted proliferation and migration of ESCC cells. Bioinformatics predictions showed that circ-TTC17 might regulate progress of ESCC by acting as a sponge for microRNAs (miRNAs).

Conclusions The results of this study demonstrate that upregulated circ-TTC17 plays a key role in promoting proliferation and migration of ESCC cells and has potential to become a novel biomarker for diagnosis, treatment, and prognosis of ESCC in the future.
\end{abstract}

Keywords ESCC $\cdot$ Circ-TTC17 $\cdot$ Upregulation $\cdot$ Proliferation $\cdot$ Migration $\cdot$ miRNA

Qian Wang and Qian Zhang contributed equally to this work.

Electronic supplementary material The online version of this article (https://doi.org/10.1007/s10620-018-5382-z) contains supplementary material, which is available to authorized users.

Hui Jin

jinzi817@live.cn

Xiufeng Cao

cxf551101@sina.com

Extended author information available on the last page of the article

\section{Introduction}

Esophageal cancer is a type of malignant tumor, and its incidence rate ranks ninth among various cancers [1]. More than 400,000 deaths from esophageal cancer occur worldwide each year, ranking sixth among all deaths from malignant tumors [2]. In China, the major pathological type of esophageal cancer is esophageal squamous cell carcinoma (ESCC). Although current medical treatments, such as surgery combined with chemotherapy, can significantly improve the diagnosis, treatment, and prognosis of ESCC patients, the 5-year survival rate is still below $16 \%$ [3]. Therefore, it is imperative to further explore the molecular mechanisms of ESCC development as well as new targets for its diagnosis and treatment. 
Circular RNAs (circRNAs) are a type of noncoding RNA without $5^{\prime}$ cap or $3^{\prime}$ tail, forming a ring structure through covalent bonds. In 1976, Sanger et al. first discovered the presence of circRNAs in plant pathogens by electron microscopy [4]. In 1991, Nigro et al. found circular transcripts in the study of DCC (a tumor suppressor gene), thus confirming the presence of circRNAs in human cells for the first time [5]. With the development of high-throughput sequencing and informatics technology, researchers have defined thousands of circRNAs in different organisms and confirmed them to be rich, conserved, and endogenous functional products [6-9] with specificity associated with species, tissues, diseases, and development stages [10-12]. Compared with traditional linear RNA, the circular structure of circRNAs is more stable and not easily digested by ribonuclease $\mathrm{R}$ (RNase R), which degrades linear RNAs [13]. More and more circRNAs have been found to play important roles in biological processes. Legnini et al. found that circ-ZNF609 has an open reading frame (ORF) and translates proteins via polyribosomes [14]. In 2013, two different research groups simultaneously demonstrated that circRNAs function as sponges for microRNAs (miRNAs) [15, 16].

The circ-TTC17 in this study was selected from the ESCC peripheral blood circRNA microarray. Its expression level was further measured in ESCC plasma samples, tissues, and cell lines by quantitative real-time polymerase chain reaction (qRT-PCR), being consistent with circRNA microarray results. Furthermore, the function of circ-TTC17 in ESCC was tested in vitro; the results showed that circ-TTC17 could improve the proliferation and migration abilities of ESCC cells. Bioinformatics analysis revealed that circ-TTC17 might regulate progress of ESCC via acting as a sponge for miRNAs.

\section{Materials and Methods}

\section{Clinical Patients and Sample Collection}

A total of 25 normal plasmas and 30 ESCC plasmas were collected from Nanjing First Hospital, Nanjing Medical University during the period from January 2015 to December 2017. A total of 25 ESCC tissues and corresponding nontumorous tissue samples were collected from ESCC patients (all from Nanjing First Hospital, Nanjing Medical University) during the period from January 2014 to December 2016. All patients did not receive radiotherapy or chemotherapy before surgery, and all tumors were accurately staged according to the tumor-node-metastasis (TNM) staging system. Written informed consent was obtained from each patient before recruitment, and the local medical ethics committee approved the study protocol.

\section{Follow-Up}

To calculate overall survival (OS), each patient were examined by a clinician every 3 months in the first year, every 6 months in the second year, and annually after the index surgery until December 2017. Date of death was obtained from inpatient and outpatient records or from patients' families through telephone follow-up.

\section{RNase $R$ and Actinomycin D Treatment}

For RNase R treatment, 2 mg total RNA was incubated for 20 min at $37{ }^{\circ} \mathrm{C}$ with or without $3 \mathrm{U} \mathrm{mg}^{-1}$ RNase R (Epicentre Technologies, Madison, WI), and the resulting RNA was purified thereafter using an RNeasy MinElute cleaning Kit (Qiagen). For actinomycin D treatment, $2 \mathrm{mg}$ total RNA was incubated at $37{ }^{\circ} \mathrm{C}$ with or without $1 \mathrm{mg}$ actinomycin D (actinomycin D, Sigma, Chengdu best reagent company), and the resulting RNA was checked separately at $0,6,12$, and $18 \mathrm{~h}$.

\section{Cell Culture and Transfection}

The ESCC cell lines used were mainly from the Shanghai Institutes for Biological Sciences, Chinese Academy of Sciences. The human normal esophageal cell line named HET1A was a generous gift from Dr. Zhihua Liu at the State Key Laboratory of Molecular Oncology, Cancer Institute, Chinese Academy of Medical Sciences (Beijing, China). siRNAs $(50 \mathrm{nM})$ targeting circ-TTC17 (si-circ-TTC17) and a negative control (NC) obtained via Lipofectamine 2000 transfection reagent (Invitrogen, Carlsbad, CA) were applied to transected KYSE30 (K30) and KYSE450 (K450) cell lines based on manufacturer instructions. All cells used in this study were cultured in Roswell Park Memorial Institute (RPMI) 1640 medium (BI, USA) containing $10 \%$ fetal bovine serum (FBS) at $37{ }^{\circ} \mathrm{C}$ in a humidified incubator containing $5 \% \mathrm{CO}_{2}$. The target sequences of circ-TTC17 siRNAs (RIBOBIO, China) were as follows: siRNA-1: 5'-TTTESCCAGTGAACACTTCCT-3'; siRNA-2 5'-ESCCA GTGAACACTTCCTCGT-3'; siRNA-3: 5'-AGTGAACAC TTCCTCGTGG-3'. Finally, the knockdown efficiency of circ-TTC17 was measured using qRT-PCR.

\section{Nucleus-Cytoplasm Fractionation}

First, $1 \times 10^{6}$ ESCC cells were washed twice with precooled PBS. The cell layer was then scraped into $500 \mu \mathrm{l}$ PBS and centrifuged at $500 \times g$ for $5 \mathrm{~min}$ at $4{ }^{\circ} \mathrm{C}$. Thereafter, the supernatant was removed and the washing step was performed twice. Finally, nuclear and cytoplasmic 
RNA from cultured ESCC cells were isolated using PARIS KIT50 RXNS (life, AM1921) according to manufacturer instructions. Corresponding mRNA was detected as a control of nuclear RNA and cytoplasmic RNA in the isolated RNA, respectively. Biotriples were used, then qRT-PCR was performed to assess the levels of circRNA and corresponding mRNA.

\section{Total RNA Isolation, Reverse Transcription, and qRT-PCR}

Total RNA from plasma, tissues, and cell lines was extracted using the Total RNA Isolation Kit (TIANGEN, China) and stored in a $-40{ }^{\circ} \mathrm{C}$ freezer. All complementary DNA (cDNAs) were obtained using the Goldenstar ${ }^{\mathrm{TM}}$ RT6 cDNA synthesis kit (TSINGKE, China) according to manufacturer instructions and stored in a $-40{ }^{\circ} \mathrm{C}$ freezer. The expression level of circ-TTC17 was measured by qRT-PCR using the following primer sequences: forward, 5'-TCCAGAGTGTGAAAACAESCCC-3'; reverse, 5'-GAGGAAGTGTTCACTESCCAAAC-3'. Glyceraldehyde 3-phosphate dehydrogenase (GAPDH) was chosen as internal control, using the positive 5'-CTGGESCCTACA CTGAESCCACC-3' to normalize the circ-TTC17 level to GAPDH, then 5'-AAGTGGTCGTTGAGGESCCAATG-3'. qRT-PCR reactions were performed using an ABI 7500 system (Applied Biosystems, Foster City, CA) and 2xT5 Fast qPCR Mix (SYBR Green I) (TSINGKE, China).

\section{Colony Formation Experiment and Cell Counting Kit-8 Proliferation (CCK-8) Assay}

In the colony formation experiment, transfected cells were seeded into six-well plates at density of 1000 cells per well and cultured in RPMI 1640 medium containing $10 \%$ FBS. After 10 days, the cells were fixed with methanol and stained with Giemsa reagent. Finally, colonies were imaged and counted.

For the CCK-8 assay, transfected cells were seeded into 96-well plates at density of 1000 cells per well, and $10 \mu \mathrm{l}$ CCK-8 solution (MCE, China) was added to each well using cells at $0,24,48,72$, and $96 \mathrm{~h}$. After treatment, plates were incubated at $37^{\circ} \mathrm{C}$ for $3 \mathrm{~h}$. Finally, cell viability was measured based on the absorbance at $450 \mathrm{~nm}$ per well. The theory behind the CCK-8 assay is that water-soluble tetrazolium salt (WST)-8 is a compound similar to 3-(4,5-dimethylthiazol2-yl)-2,5-diphenyltetrazolium bromide (MTT) that, in the presence of an electron-coupled reagent, can be reduced to some orange-form formazan by some dehydrogenase in the mitochondria; the more the cells proliferate, the darker the color; the greater the cytotoxicity, the lighter the color.

\section{Wound Healing Assay}

KYSE30 and KYSE450 cells were cultured in six-well plates and scraped using the fine end of $200-\mu$ l pipette tips (at $0 \mathrm{~h}$ ). Cell migration was photographed in 10 high-power fields at 0 and $24 \mathrm{~h}$ after injury. Remodeling was measured as the decrease in the distance across the induced injury, normalized to the $0 \mathrm{~h}$ control and expressed as relative migration.

\section{Statistical Analysis}

Data are shown as mean \pm standard deviation (SD). All statistical analyses were carried out using SPSS 18.0 statistical software and GraphPad Prism 6 using Student's $t$ test or oneway analysis of variance (ANOVA). For all results, $P<0.05$ was considered statistically significant.

\section{Results}

\section{Source and Biological Structure of Circ-TTC17}

A high-throughput human circRNA microarray was conducted using plasma samples from 10 ESCC patients, including 5 patients with T3NOM0 (case 1) and another 5 patients with T3N1-3M0 (case 2), as well as 5 normal plasma samples (control), to assess differences in the circRNA expression profile in ESCC. The correlation diagram shown in Supplementary Fig. 1A intuitively presents the similarity between samples. When the biological replicates of the sample was $\geq 3$, a volcanogram (volcano plot) was drawn, together with the two factors $(P$ value and fold change) obtained from the difference analysis, to show the significant difference between the two groups of samples (Fig. 1a). A total of 10 circRNAs were selected and further tested in the plasma of 10 patients with ESCC and 10 normal plasmas. The results showed that the difference in expression of circ-TTC17 was the most notable, and it was thus chosen for further study (Supplementary Fig. 1B). The primer sequences of these circRNAs are listed in Supplementary Table 1.

Circ-TTC17 was formed from exon 6 to 16 of the gene TTC17 and was located in chr 1: 43418258-43436326; the Sanger sequencing of the qRT-PCR product was comparable to the sequence of the splicing junction of circ-TTC17 (Supplementary Fig. 1C). Agarose gel electrophoresis was used to verify the specificity of circ-TTC17 (Supplementary Fig. 1D). Random or oligo (dT)18 primers were used in the reverse-transcription experiments (Supplemental Fig. 1E). The results of actinomycin $\mathrm{D}$ and RNase $\mathrm{R}$ exonuclease treatment showed that circ-TTC17 was resistant to actinomycin D and RNase R exonuclease digestion (Supplementary Fig. 2A, B). 


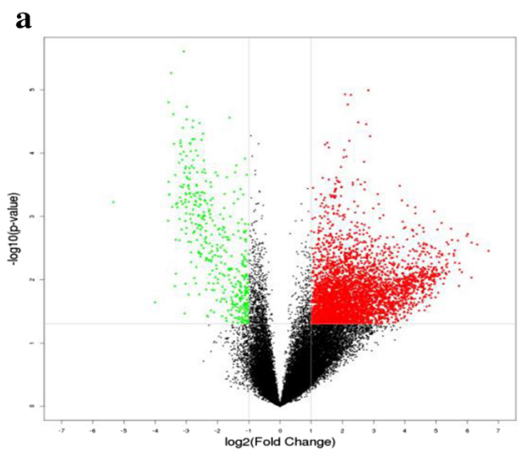

b
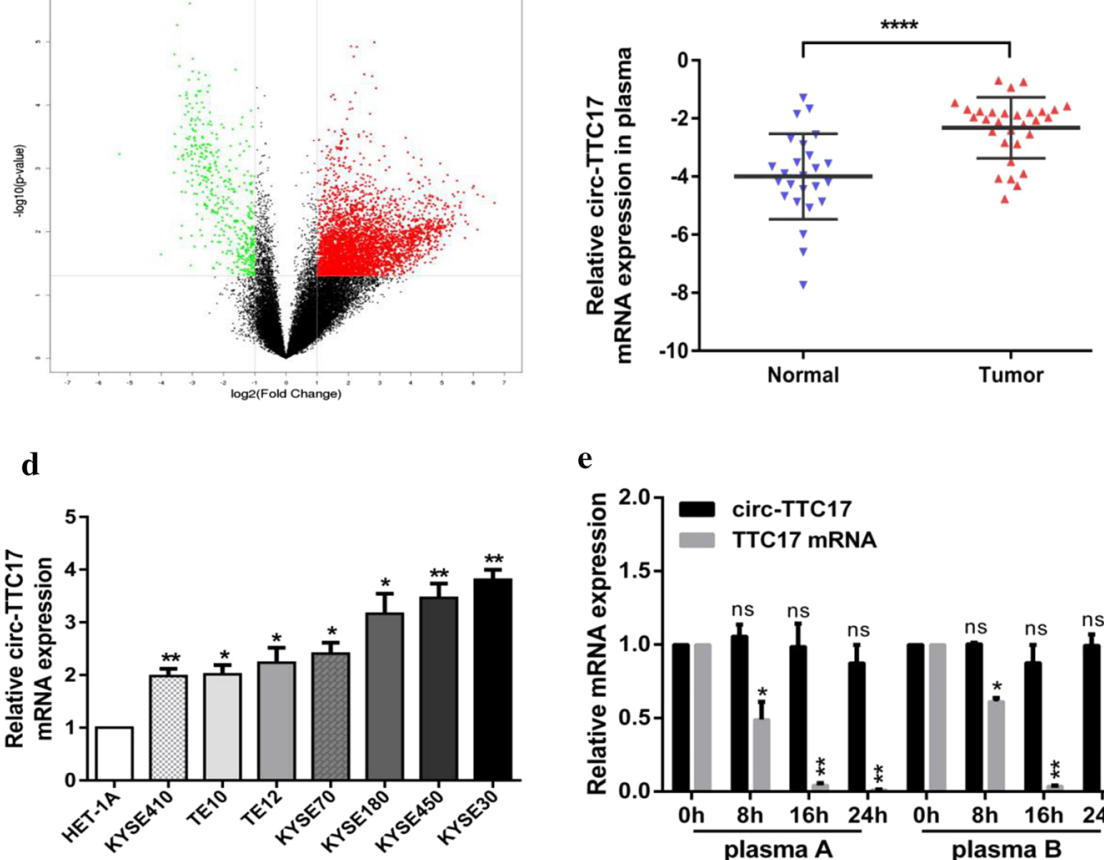

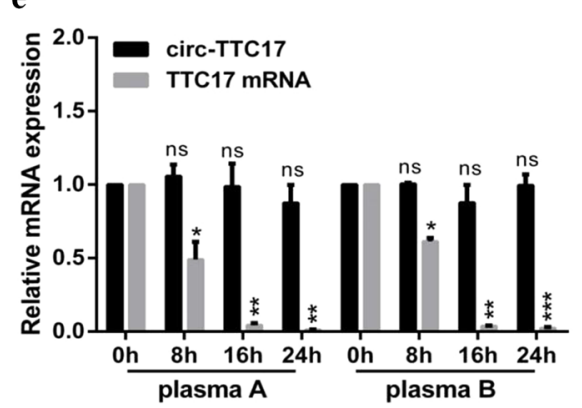

c

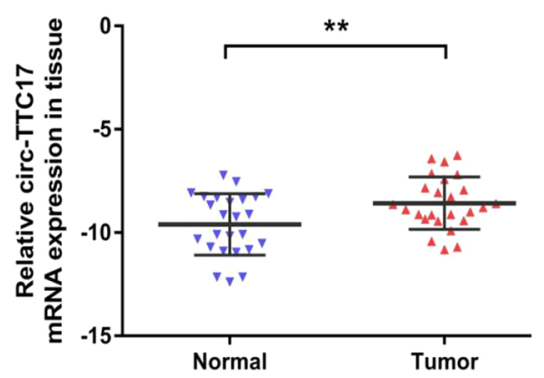

f

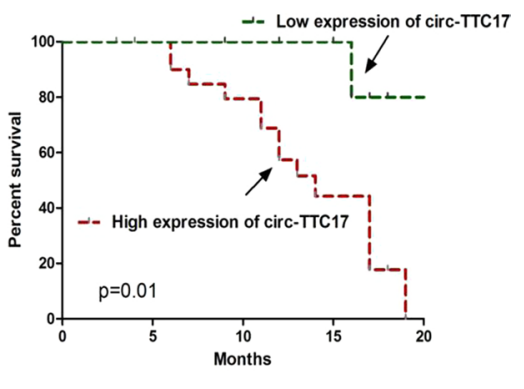

Fig. 1 Circ-TTC17 expression in clinical samples of ESCC patients and ESCC cell lines. a Volcano plot showing the different expressions of circRNAs in case 1 versus normal control group. Among them, those marked with red were upregulated genes, those marked with green were downregulated genes, while those marked with black showed no significant differences. The closer the points in the figure to the upper left or upper right corner, the more significant the difference. b The expression level of circ-TTC17 was significantly higher in ESCC plasmas than in normal plasmas. $\mathbf{c}$ The expression level of

\section{Circ-TTC17 Expression in ESCC Patients and ESCC Cell Lines}

To confirm the tendency for higher expression of circTTC17 in ESCC plasma, we expanded the number of ESCC plasma samples, obtaining consistent results (Fig. 1b). To confirm that circ-TTC17 expression in tissues is consistent with that in plasma, we further studied it in 25 paired primary cancerous and adjacent noncancerous tissues from ESCC patients. The results showed that the expression level of circ-TTC17 was significantly higher than in adjacent noncancerous tissue (Fig. 1c). The expression level of circTTC17 in ESCC cell lines was examined, revealing that circTTC17 expression in ESCC cells was significantly higher than that in HET-1A (Fig. 1d). Meanwhile, we verified the different stability of circ-TTC17 and TTC17 mRNA in two ESCC plasma samples (Fig. 1e).

We used the receiver operating characteristic (ROC) curve to investigate the diagnostic value of circ-TTC17 in distinguishing ESCC from normal plasma. The area under the ROC curve (AUC) was 0.8200, as shown in Supplementary Fig. 2C, and the cutoff value of circ-TTC17
circ-TTC17 was significantly higher in ESCC tissues than in adjacent noncancerous tissues. d Expression of circ-TTC17 in ESCC cell lines was significantly higher than in normal cell line HET-1A. e The stability of circ-TTC17 and TTC17 mRNA was verified in two plasma samples from different ESCC patients. f Kaplan-Meier overall survival curve, revealing that patients with higher circ-TTC17 expression showed reduced survival time. $* P<0.05 ; * * P<0.01$; $* * * P<0.001$. ns not significant

was -2.548 with specificity of $88.00 \%$ and sensitivity of $73.33 \%$. The association of upregulation of circ-TTC17 in ESCC patients with their clinicopathological features was also analyzed. As shown in Table 1, circ-TTC17 in plasma was not aberrantly associated with age, gender, differentiation, tumor size, or common clinical biomarkers including AFP, CEA, and SCCA in patients with ESCC. However, the expression level of circ-TTC17 was positively associated with TNM stage $(P=0.012)$ and lymphatic metastasis $(P=0.0006)$. Kaplan-Meier survival analysis and log-rank tests using patient postoperative survival were performed to further evaluate the correlation between circ-TTC17 expression and prognosis of patients with ESCC. The results revealed that ESCC patients with higher level of circ-TTC17 expression had significantly shorter survival time than those with lower level of circ-TTC17 expression (Fig. 1f). Furthermore, univariate analysis indicated that relative circ-TTC17 expression level $(P=0.040)$, TNM stage $(P=0.033)$, and lymphatic metastasis $(P=0.039)$ were respectively determined as prognostic indicators for the overall survival rate of ESCC patients. However, multivariate analysis of variance showed that 
Table 1 Clinicopathological characteristics and expression of circTTC17

\begin{tabular}{|c|c|c|c|c|}
\hline Variable & Case & Low expression & $\begin{array}{l}\text { High } \\
\text { expres- } \\
\text { sion }\end{array}$ & $P$ value \\
\hline Age (years) & & & & 0.715 \\
\hline$\geq 65$ & 17 & 5 & 12 & \\
\hline$<65$ & 13 & 3 & 10 & \\
\hline Gender & & & & 0.087 \\
\hline Female & 8 & 4 & 4 & \\
\hline Male & 22 & 4 & 18 & \\
\hline Size & & & & 0.221 \\
\hline$\geq 3 \mathrm{~cm}$ & 21 & 7 & 14 & \\
\hline$<3 \mathrm{~cm}$ & 9 & 1 & 8 & \\
\hline Differentiation & & & & 0.430 \\
\hline Low & 11 & 2 & 9 & \\
\hline High/intermediate & 19 & 6 & 13 & \\
\hline TNM stage & & & & $0.012 *$ \\
\hline I-II & 15 & 7 & 8 & \\
\hline III-IV & 15 & 1 & 14 & \\
\hline Lymph node metastasis & & & & $0.006^{*}$ \\
\hline No & 18 & 8 & 10 & \\
\hline Yes & 12 & 0 & 12 & \\
\hline CEA & & & & 0.791 \\
\hline High expression & 10 & 3 & 7 & \\
\hline Low expression & 20 & 5 & 15 & \\
\hline AFP & & & & 0.221 \\
\hline High expression & 9 & 1 & 8 & \\
\hline Low expression & 21 & 7 & 14 & \\
\hline SCCA & & & & 0.436 \\
\hline High expression & 8 & 3 & 5 & \\
\hline Low expression & 22 & 5 & 17 & \\
\hline
\end{tabular}

$* P<0.05$

$C E A$ carcinoembryonic antigen, $A F P \alpha$-fetoprotein, SCCA squamous cell carcinoma antigen

only tumor size was an independent marker for assessing prognosis of ESCC patients (Table 2).

\section{Circ-TTC17 Alters Proliferation and Migration of ESCC}

To investigate the potential role of circ-TTC17 in ESCC cells, KYSE30 cells were transfected with si-circ-TTC17 or si-NC using Lipofectamine 2000 as transfection reagent. After $48 \mathrm{~h}$ of incubation, circ-TTC17 expression was effectively knocked down in KYSE30 cells, as shown in Supplementary Fig. 2D, and si-circ-TTC17-3 showed the highest knockdown efficiency, being further selected for subsequent experiments. The colony formation experiment and CCK- 8 assay showed that, after knockdown of circ-TTC17, the proliferation ability of KYSE30 and KYSE450 cells was significantly decreased (Fig. 2a, b). The scratch-wound assay in KYSE30 and KYSE450 cells demonstrated that suppression of circ-TTC17 exhibited a lower scratch closure rate compared with control groups treated with si-NC, respectively (Fig. 2c). However, the invasive ability of ESCC cells showed no obvious difference under the effect of circ-TTC17.

\section{Prediction of circRNA-miRNA-mRNA Associations and Bioinformatics Analysis}

To further explore the location of circ-TTC17 in ESCC cells, we performed a nuclear and cytoplasm separation experiment and found that circ-TTC17 was mainly located in the cytoplasm while its source gene TTC17 was mainly in the nucleus (Supplemental Fig. 3A). We downloaded the available datasets from doRiNA (http://dorina.mdc-berlin.de) and acquired the AGO2-binding sites of the circ-TTC17 genomic region, which revealed that circ-TTC17 had a high degree of AGO2 occupancy (Supplemental Fig. 3B).

To identify the miRNAs binding to circ-TTC17, we predicted a circRNA-miRNA-mRNA interaction network using the CircInteractome, starBase, and TargetScan databases. A total of 20 miRNAs (e.g., miR-153, miR-217, miR-224, and miR-370) and corresponding target mRNAs were predicted to have an interaction with circ-TTC17 in this study (Supplementary Fig. 3C).

\section{Discussion}

Kaplan-Meier survival analysis revealed that ESCC patients with higher expression level of circ-TTC17 had significantly shorter survival time than those with lower expression level of circ-TTC17. These results reveal that preoperative detection in patients' plasma could be used to diagnose ESCC as well as for comprehensive assessment of clinical stage, lymph node metastasis, and prognosis. Of course, one of the major drawbacks of this experiment is that it was not possible to further detect expression of circ-TTC17 in other cancers such as gastric, liver, or colorectal cancer, to rule out their interference with the detection of circ-TTC17.

The in vitro results show that knockdown of circ-TTC17 significantly inhibited proliferation and migration of ESCC cells, indicating that circ-TTC17 could accelerate progression of ESCC. Although there is relatively little research on circRNAs and ESCC, this is not the first study to investigate their relation. Xia et al. [17] characterized a novel circRNA termed hsa_circ_0067934 in ESCC tumor tissues and cell lines, finding that high expression level of hsa_circ_0067934 was associated with poor differentiation and TNM stage I-II. Silencing of hsa_circ_0067934 in vitro by siRNA inhibited proliferation and migration of ESCC cells and blocked cell 
Table 2 Univariate and multivariate analyses for overall survival

\begin{tabular}{lrlllllr}
\hline Variable & \multicolumn{1}{c}{$B$} & SE & Wald & Sig. & Exp. & Lower limit & Upper limit \\
\hline Univariate & & & & & & & \\
Age (years) & -0.148 & 0.559 & 0.071 & 0.790 & 0.862 & 0.289 & 2.576 \\
Gender & 0.504 & 0.587 & 0.736 & 0.391 & 1.655 & 0.523 & 5.233 \\
Size & -0.923 & 0.572 & 2.603 & 0.107 & 0.397 & 0.129 & 1.219 \\
Differentiation & -0.030 & 0.531 & 0.003 & 0.955 & 0.970 & 0.343 & 2.747 \\
TNM stage & 1.378 & 0.646 & 4.556 & $0.033^{*}$ & 3.968 & 1.119 & 14.065 \\
Lymph node metastasis & -1.101 & 0.535 & 4.240 & $0.039^{*}$ & 0.333 & 0.117 & 0.948 \\
CEA & 0.603 & 0.653 & 0.853 & 0.356 & 1.828 & 0.508 & 6.573 \\
AFP & 0.322 & 0.593 & 0.295 & 0.587 & 1.380 & 0.431 & 4.414 \\
SCCA & 0.206 & 0.659 & 0.098 & 0.754 & 1.229 & 0.338 & 4.474 \\
Circ-TTC17 expression & 2.136 & 1.038 & 4.234 & $0.040^{*}$ & 8.466 & 1.107 & 64.758 \\
Multivariate & & & & & & & \\
Age (years) & 0.070 & 0.831 & 0.007 & 0.933 & 1.073 & 0.211 & 5.462 \\
Gender & -0.412 & 1.001 & 0.169 & 0.681 & 0.662 & 0.093 & 4.712 \\
Size & -2.340 & 1.091 & 4.604 & $0.032^{*}$ & 0.096 & 0.011 & 0.817 \\
Differentiation & 0.407 & 0.747 & 0.298 & 0.585 & 1.503 & 0.348 & 6.494 \\
TNM stage & -0.133 & 1.114 & 0.014 & 0.905 & 0.875 & 0.099 & 7.771 \\
Circ-TTC17 expression & 1.354 & 1.375 & 0.969 & 0.325 & 3.873 & 0.261 & 57.364 \\
Lymph node metastasis & -1.131 & 0.967 & 1.368 & 0.242 & 0.323 & 0.049 & 2.147 \\
CEA & 1.429 & 1.048 & 1.860 & 0.173 & 4.176 & 0.535 & 32.584 \\
AFP & 0.834 & 0.710 & 1.382 & 0.240 & 2.303 & 0.573 & 9.258 \\
SCCA & 0.607 & 1.087 & 0.312 & 0.576 & 1.836 & 0.218 & 15.461 \\
\hline
\end{tabular}

$* P<0.05$

cycle progression. These findings identify a novel potential biomarker and therapeutic target of ESCC.

The emerging roles of RNA-RNA crosstalk, as part of a complex posttranscriptional regulatory circuit, have been implicated in human development and disease [18]. Recently, SRY, the testis-specific circRNA, was validated to act as an miR-138 sponge, with ceRNA activity [16], suggesting that these circRNAs may play significant roles in regulatory RNA networks. An antisense transcript to CDR1, termed CDR1as or circRNA sponge for miR-7, is highly expressed in human and mouse brain. CDR1as contains $>70$ selectively conserved miRNA target sites and suppresses miR-7 activity, resulting in enhanced expression of miR-7 targets [19]. As a result of their high expression level and stability, circRNAs with ceRNA function may be particularly effective modulators of crosstalk in linear ceRNAs. In the study presented herein, a nuclear-cytoplasm separation experiment confirmed that circ-TTC17 was mainly located in the cytoplasm while its source gene TTC17 was located in the nucleus. Datasets from doRiNA revealed that circTTC17 had a high degree of AGO2 occupancy. A total of 20 microRNAs and corresponding target mRNAs were predicted to have an interaction with circ-TTC17 in this study. However, the correlation between circ-TTC17 and miRNAs needs to be further confirmed, including luciferase reporter experiments to verify the regulatory site, and qPCR tests to confirm the expression correlation. Although more and more circular RNAs with known functions have been studied and reported, a large number of circular RNAs in tumors, especially ESCC, remain unknown. Therefore, more studies must be done to confirm the roles and molecular mechanisms of circRNAs in ESCC.

\section{Conclusions}

The results of this study show that circ-TTC17 is significantly upregulated in ESCC plasma, tissues, and cells compared with normal controls, having advantages over linear TTC17 as a clinical biomarker in ESCC. In addition, circTTC17 promotes proliferation and migration of ESCC cells by acting as a sponge for miRNAs; however, the precise mechanism in this functional process deserves further exploration and validation.

Funding This work was supported by the National Natural Science Foundation of China (NSFC) (nos. 81372656, 81672423), Jiangsu Natural Science Foundation (BK20151292), Jiangsu Provincial Medical Innovation Team of the Project of Invigorating Health Care through Science, Technology, and Education, 333 Project Foundation of Jiangsu Province (no. BRA2014355), and Six Talent Peaks Project in Jiangsu Province (WSN-084). 
a

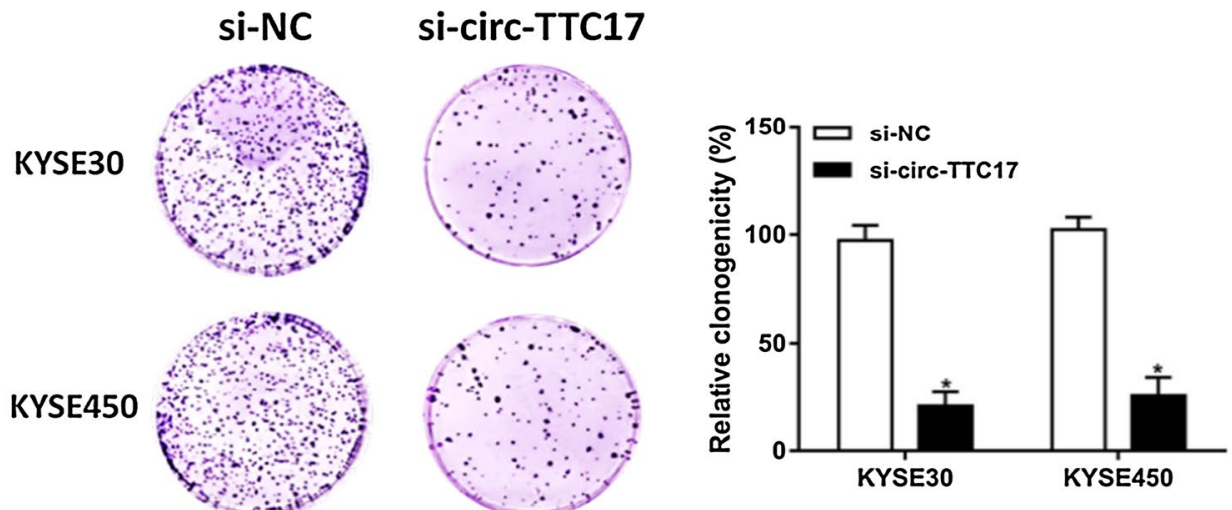

b
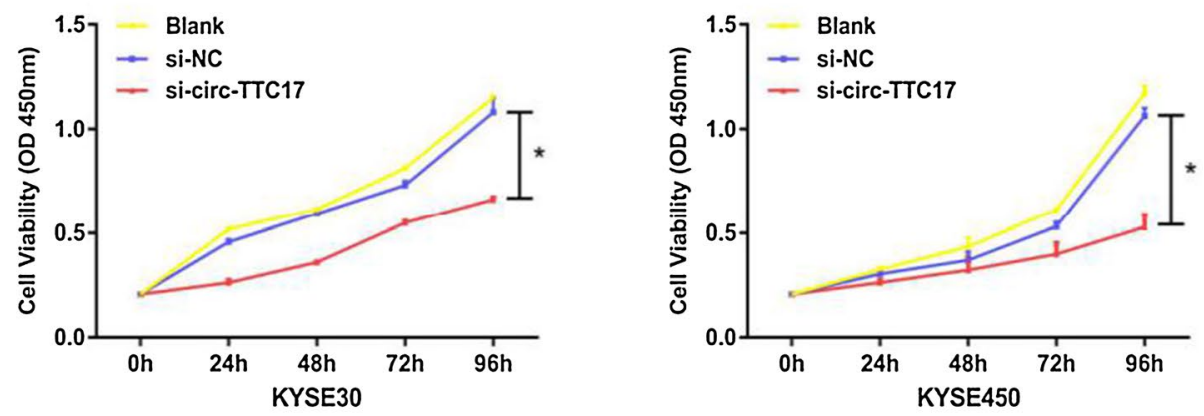

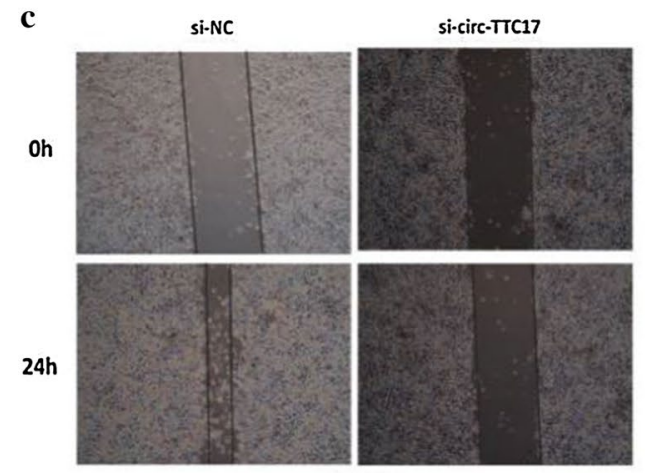

KYSE30

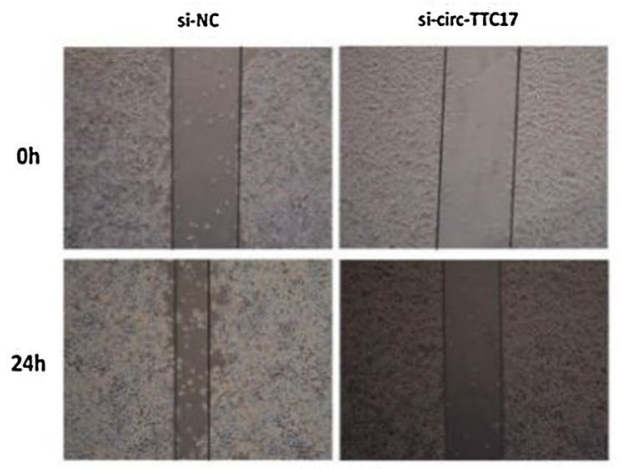

KYSE450

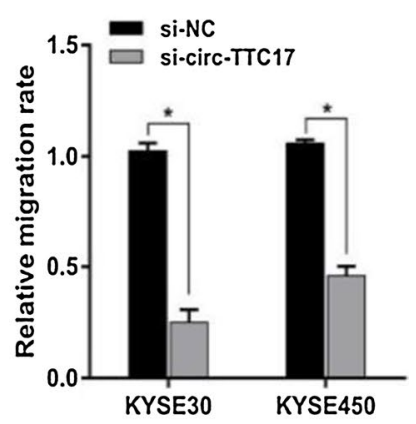

Fig. 2 Functions of circ-TTC17 in progression of ESCC cells. a Colony formation experiment and b CCK-8 assay showed that knockdown of circ-TTC17 inhibited cell proliferation significantly in
KYSE30 and KYSE450 cells. c Knockdown of circ-TTC17 inhibited cell migration significantly in KYSE30 and KYSE450 cells. $* P<0.05$

\section{Compliance with ethical standards}

Conflict of interest The authors declare that they have no competing interests.

Consent for publication We obtained consent from each patient who participated in this study. The consent forms are available upon request.

Open Access This article is distributed under the terms of the Creative Commons Attribution-NonCommercial 4.0 International License (http://creativecommons.org/licenses/by-nc/4.0/), which permits any noncommercial use, distribution, and reproduction in any medium, provided you give appropriate credit to the original author(s) and the source, provide a link to the Creative Commons license, and indicate if changes were made.

Ethical approval Use of the human cancer tissues in this study was approved by the Ethics Committee of Nanjing First Hospital, Nanjing Medical University. 


\section{References}

1. Miller KD, Siegel RL, Lin CC, et al. Cancer treatment and survivorship statistics, 2016. CA Cancer J Clin. 2016;66:271-289.

2. Torre LA, Bray F, Siegel RL, et al. Global cancer statistics, 2012. CA Cancer J Clin. 2015;65:87-108.

3. Chen YH, Li SH, Chiu YC, et al. Comparative study of esophageal stent and feeding gastrostomy/jejunostomy for tracheoesophageal fistula caused by esophageal squamous cell carcinoma. Plos One. 2012;7:e42766.

4. Sanger HL, Klotz G, Riesner D, et al. Viroids are single stranded covalently closed circular RNA molecules existing as highly base-paired rod-like structures. Proc Natl Acad Sci USA. 1976;73:3852-3856.

5. Nigro JM, Cho KR, Fearon ER, et al. Scrambled exons. Cell. 1991;64:607-613.

6. Salzman J, Gawad C, Wang PL, et al. Circular RNAs are the predominant transcript isoform from hundreds of human genes in diverse cell types. PLoS One. 2012;7:e30733.

7. Danan M, Schwartz S, Edelheit S, et al. Transcriptomewide discovery of circular RNAs in Archaea. Nucleic Acids Res. 2012;40:3131-3142.

8. Jeck WR, Sorrentino JA, Wang K, et al. Circular RNAs are abundant, conserved, and associated with ALU repeats. RNA. 2013;19:141-157.

9. Salzman J, Chen RE, Olsen MN, et al. Cell-type specific features of circular RNA expression. PLoS Genet. 2013;9:e1003777.

10. Dong R, Ma XK, Chen LL, et al. Increased complexity of circRNA expression during species evolution. RNA Biol. 2017;14:1064-1074.

\section{Affiliations}

\section{Qian Wang ${ }^{1} \cdot$ Qian Zhang ${ }^{1,2} \cdot$ Handong Sun ${ }^{1} \cdot$ Weiwei Tang ${ }^{3} \cdot$ Lili Yang $^{4} \cdot$ Zhipeng Xu $^{2} \cdot$ Zhihua Liu $^{5} \cdot$ Hui Jin $^{6,7,8}$. Xiufeng $\mathrm{CaO}^{1,9}$}

1 Department of Oncology Surgery, Nanjing First Hospital, Nanjing Medical University, Nanjing, China

2 Department of Oncology Surgery, Nanjing SIR RUN RUN Hospital, Nanjing Medical University, Nanjing, China

3 Department of General Surgery, Nanjing First Hospital, Nanjing Medical University, Nanjing, Jiangsu, China

4 Department of Oncology, Second Hospital of Nanjing, Nanjing Medical University, Nanjing, China

5 State Key Laboratory of Molecular Oncology, National Cancer Center/Cancer Hospital, Chinese Academy of Medical Sciences and Peking Union Medical College, Beijing, China
11. Werfel S, Nothjunge S, Schwarzmayr T, et al. Characterization of circular RNAs in human, mouse and rat hearts. J Mol Cell Cardiol. 2016;98:103-107.

12. Chen W, Schuman E. Circular RNAs in brain and other tissues: a functional enigma. Trends Neurosci. 2016;39:597-604.

13. Hitoshi S, Zuo Y, Wang J, et al. Characterization of RNase R-digested cellular RNA source that consists of lariat and circular RNAs from pre-mRNA splicing. Nucleic Acids Res. 2006;34:e63.

14. Legnini I, Di Timoteo G, Rossi F, et al. Circ-ZNF609 is a circular RNA that can be translated and functions in myogenesis. Mol Cell. 2017;66:22-37.

15. Memczak S, Jens M, Elefsinioti A, et al. Circular RNAs are a large class of animal RNAs with regulatory potency. Nature. 2013;495:333-338.

16. Hansen TB, Jensen TI, Clausen BH, et al. Natural RNA circles function as efficient microRNA sponges. Nature. 2013;495:384-388.

17. Xia W, Qiu M, Chen R, et al. Circular RNA has_circ_0067934 is upregulated in esophageal squamous cell carcinoma and promoted proliferation. Sci Rep. 2016;18:35576.

18. Ebert MS, Neilson JR, Sharp PA. MicroRNA sponges: competitive inhibitors of small RNAs in mammalian cells. Nat Methods. 2007;4:721-726.

19. Zhang X, Yang D, Wei Y. Overexpressed CDR1as functions as an oncogene to promote the tumor progression via miR-7 in nonsmall-cell lung cancer. Oncotargets Ther. 2018;11:3979-3987.
6 Department of Hematology, The First Affiliated Hospital of Nanjing Medical University, Jiangsu Province Hospital, Nanjing, China

7 Key Laboratory of Hematology of Nanjing Medical University, Nanjing, China

8 Collaborative Innovation Center for Cancer Personalized Medicine, Nanjing, China

9 Department of Thoracic Surgery, Taikang Xianlin Drum Tower Hospital, School of Medicine, Nanjing University, Nanjing, China 